\title{
A CATALOG OF SPECTROSCOPICALLY IDENTIFIED WHITE DWARFS
}

\author{
GEORGE P. MCCOOK and EDWARD M. SION \\ Department of Astronomy and Astrophysics, Villanova University
}

\begin{abstract}
A catalog of 2249 white dwarfs which have been identified spectroscopically is presented complete through 1996 April. This compilation is the fourth edition of the Villanova Catalog of Spectroscopically Identified White Dwarfs. For each degenerate star, the following data entries with references are provided: (1) a catalog coordinate designation or WD number, in order of right ascension; (2) the right ascension and declination for epoch 1950.0; (3) the spectral type based upon the new system;(4) a catalog symbol denoting binary membership; (5) a list of most names known to exist for a given star; (6) proper motion and position angle; (7) broad-band $U B V$ photometry, $V, B-V, U-B$; (8) multichannel spectrophotometry, $V(M C), g-r$; (9) Strömgren narrow-band photometry $y$, $b-y, u-b ;(10)$ an absolute visual magnitude based upon the best available color-magnitude calibration or trigonometric parallax; (11) the observed radial velocity uncorrected for gravitational redshift or solar motion; and (12) the trigonometric parallax with mean error when available. A Notes section for unusual or peculiar stars and a coded Reference Key alphabetized by the first author's last name are presented, as well as an expanded table cross-referencing all names to catalog WD number. An introduction and full descriptions of the entries are provided in the text.
\end{abstract}

\section{INTRODUCTION}

The first edition of this catalog was published privately in 1977 May (McCook \& Sion 1977) as Villanova University Observatory Contribution No. 2 and contained less than 600 degenerate stars. The preface to that edition provided a succinct rationale of our motivation for preparing the catalog and is presented here verbatim:

The number of newly discovered white dwarfs has increased substantially since the appearance of the first comprehensive list by Eggen $\& 3$ Greenstein in 1965. The newly observed objects when added to the earlier lists comprise a sample approaching six hundred stars. New spectroscopic, spectrophotometric and photometric observations are being obtained by several active groups throughout the world. The volume of data is enormous and much of it is dispersed throughout the literature. Some confusion exists with nomenclature especially regarding multiple names. The volume of data and the nature of its accessibility in the literature strongly suggested the need for a general comprehensive white dwarf catalogue. While there is clearly no substitute 
for using the primary reference literature, it may prove useful in certain applications for both observers and theoreticians to have the data collected into one catalogue. We apologize in advance for the inevitable errors in a catalogue of this size and welcome corrections and additions being brought to our attention for inclusion in possible future editions.

Since the appearance of the first edition of this catalog (McCook \& Sion 1977), the number of newly identified spectroscopic degenerates has more than quadrupled. Building upon the identification of several hundred new spectroscopic degenerates with the $5.1 \mathrm{~m}$ Hale reflector by Jesse Greenstein and with the Palomar-Green north galactic pole survey of hot white dwarfs by Green, Schmidt, \& Liebert (1986), the past two decades have seen over 1500 new spectroscopically identified white dwarfs discovered in several large scale color-select, objective prism and proper motion-selected surveys. The sources of new degenerates are principally the following surveys: the APM Proper Motion Project (D. Evans), the Case Low Dispersion Objective Prism Survey (P. Pesch, M. Wagner and co-workers), the Edinburgh-Cape Survey (D. O'Donoghue, Kilkenny, Stobie, and co-workers), the EUVE Sample (S. Bowyer, S. Vennes and co-workers), the Hamburg-Schmidt Survey (U. Heber and coworkers), the HK Objective Prism Survey (T. Beers and co-workers), the KISO UV-Excess Survey (G. Wegner and co-workers), Kitt Peak-Downes Survey (R. Downes and co-workers), the Large Bright Quasar Survey (C. Foltz and coworkers), Luyten/Giclas Common Proper Motion Binary Survey (T. Oswalt and co-workers), the Montreal-Cambridge-Tololo Survey (G. Fontaine, F. Wesemael and co-workers), the Palomar-Green Survey (R. Green, J.Liebert and co-workers), the ROSAT-detected Sample (M. Barstow, T. Fleming and coworkers), and the UVX COSMOS Survey (B. Boyle and co-workers). The specific references to these sources are found in the Reference Key to Table 3, which appears at the end of the paper.

This enlarged sample of spectroscopically identified degenerates is accompanied by an expanded database of new photometric colors, parallaxes, proper motions, and radial velocities, as well as expanded names entries and notes section. In addition, a superbly illustrated, comprehensive spectroscopic atlas of all of the identified types of white dwarf spectra by Wesemael et al. 1993 is available for easy reference to the types of spectra contained in this fourth edition catalogue.

We have excluded references to finding charts in all previous editions and continue this practice in the present edition. However, at the time of this writing, a comprehensive on-line finding chart atlas is in preparation by Tom Marsh, Chris Moran and collaborators in the United Kingdom. Moreover we refer the user to an atlas of finding charts for EUVE sources published by M. Shara et al. (1997). 


\section{Selection of the Data: Entry Changes Since the Third Edition}

As in the first, second and third editions, we have attempted in this fourth edition to include only white dwarfs with published spectroscopic identification. In a very few cases, colors and motions strongly indicate a white dwarf, but no spectral class has as yet been assigned. We have included white dwarfs that are components of essentially noninteracting binaries (e.g., Roche lobe-detached, short and long-period systems) whose detected photospheric spectra are classifiable. A few examples illustrate the morphological range of such binary systems: HZ 9, Sirius B, HZ 43, and the DA component of the barium star Zeta Cet. We have attempted to include all data published or released to us in preprint form prior to 1996 April. On the basis of input received from colleagues throughout the world, content changes have been introduced in the fourth edition that we hope will help to maximize its scientific usefulness.

\subsection{Spectroscopic Classification Modifications}

The revised white dwarf spectral classification system presented by Sion et al. (1983) and implemented in the third edition (McCook \& Sion 1987) is presented in table 1. This system has undergone some further modifications, discussed most recently by Sion (1996), Wesemael et al. (1993) and by Liebert \& Sion (1994) in light of both the increasing accuracy of derived atmospheric parameters for degenerate stars and the discovery of degenerate stars in which elements heavier than helium are the dominant photospheric constituent. A number of these modifications were originally discussed by Sion et al. (1983) but never implemented in practice. 
TABLE 1

DEFINITION OF PRIMARY SPECTRAL SYMBOLS

\begin{tabular}{|c|c|}
\hline Spectral Type & Characteristics \\
\hline DA & Only Balmer lines; no He I or metals present \\
\hline DB .................. & He I lines; no $\mathrm{H}$ or metals present \\
\hline DC .... & $\begin{array}{l}\text { Continuous spectrum, no lines deeper than } 5 \% \\
\text { in any part of the electromagnetic spectrum }\end{array}$ \\
\hline $\mathrm{DO}$ & He II strong; He I or H present \\
\hline DZ & Metal lines only; no $\mathrm{H}$ or He lines \\
\hline DQ & $\begin{array}{l}\text { Carbon features, either atomic or molecular in } \\
\text { any part of the electromagnetic spectrum }\end{array}$ \\
\hline $\mathrm{P}$ & - magnetic white dwarfs with detectable polarization \\
\hline $\mathrm{H}$ & - magnetic white dwarfs without detectable polarization \\
\hline $\mathrm{X}$ & - peculiar or unclassifiable spectrum \\
\hline $\mathrm{E}$ & - emission lines are present \\
\hline$?$ & - uncertain assigned classification; a colon (:) may also be used \\
\hline $\mathrm{V}$ & - optional symbol to denote variability \\
\hline
\end{tabular}

\subsubsection{Refined (Decimal Half Integer and Double Digit) Temperature Indices}

Because of the importance of temperature as a direct luminosity and age indicator in white dwarfs and the fact that white dwarfs span enormous ranges of $\mathrm{T}_{\text {eff }}$ (e.g., the H-rich white dwarfs span a temperature range from $170,000 \mathrm{~K}$ to $4500 \mathrm{~K}$ !), a temperature index was adopted by Sion et al. (1983) defined as $10 \times \theta_{\text {eff }}\left(=50400 / T_{\text {eff }}\right)$. However, the limitations of the temperature index in the Sion et al. (1983) system become obvious at the lowest $\left(\mathrm{T}_{\text {eff }}<5040 \mathrm{~K}\right)$ and highest surface temperatures $\left(\mathrm{T}_{e f f}>50400 \mathrm{~K}\right)$ when it no longer provides the needed indicial temperature discrimination. Indeed with the increasing accuracy of derived white dwarf temperatures (and gravities) using ever more sophisticated LTE and NLTE synthetic spectra codes with improved atomic physics, a number of advantageous refinements to the temperature index suggest themselves (Liebert \& Sion 1994).

The use of a half-integer temperature index for white dwarfs with $\mathrm{T}_{\text {eff }}<$ $50,400 \mathrm{~K}$, allows for refined temperature classification with the possibility of even finer decimal sub-divisions as temperatures become increasingly more accurate. Thus for example the DA sequence would extend from DA.5, DA1, DA1.5, DA2, DA2.5 ....DA13. A DA2 star would have a temperature in the range $22,400 \mathrm{~K}$ to $28,800 \mathrm{~K}$ while a DA2.5 would have $\mathrm{T}_{\text {eff }}$ in the range $18,327 \mathrm{~K}$ to $22,400 \mathrm{~K}$. Similary the sequence of DB stars would extend from DB2, DB2.5, 
DB3, DB3.5...... A DB2 star would have a temperature in the range $22,400 \mathrm{~K}$ to $28,800 \mathrm{~K}$ while a DB2.5 would have $\mathrm{T}_{\text {eff }}$ in the range $18,327 \mathrm{~K}$ to $22,400 \mathrm{~K}$. The clear advantage of a decimal half-integer over the plus symbol ("+") is that further subdivision is possible as temperatures become even more accurate. The temperature index for the coolest degenerates is extended to double digits down to $\theta=13$, as originally proposed in Sion et al. (1983) and implemented, for example, by Greenstein (1986). For the hottest degenerates, we adopt a scheme (Liebert \& Sion 1994) whereby a zero temperature index is circumvented by opting for a decimal double digit theta index $(\theta<1.0)$. Thus, the hottest white dwarfs $(\log g \geq 7)$ are assigned a temperature index of decimal 9 to decimal 1 (.9 to .1). For example a degenerate star with $\mathrm{T}_{e f f}=50,400 \mathrm{~K}$ would have a temperature index of 1.0 while a star with $\mathrm{T}_{\text {eff }}=200,000 \mathrm{~K}$ (hotter than any presently known degenerate with $\log g \geq 7$ ) would have a temperature index of .25. Note that there is never a zero before the decimal point. Its use is prohibited because (a) the inevitable confusion with the hot primary spectral type of $\mathrm{O}$ is avoided (the distinction between zero and $\mathrm{O}$ is no longer obvious in computer operating systems) and (b) a zero index is unphysical since such an index can never occur. The range of temperatures corresponding to each half-integer spectral index is listed in table 2. 
Table 2

\begin{tabular}{lcc|}
\hline \multicolumn{3}{c}{ TEMPERATURE INDEX RANGES } \\
Sp.Type & $T_{\text {eff }}$ Range $(\mathrm{K})$ & $\Theta_{\text {eff }}$ Range \\
\hline DA.25 & 200,000 & \\
DA.5 & 100,800 & \\
DA1 & $40,320-67,200$ & $1.25-0.75$ \\
DA1.5 & $28,800-40,320$ & $1.75-1.25$ \\
DA2 & $22,400-28,800$ & $2.25-1.75$ \\
DA2.5 & $18,327-22,400$ & $2.75-2.25$ \\
DA3 & $15,507-18,327$ & $3.25-2.75$ \\
DA3.5 & $13,340-15,507$ & $3.75-3.25$ \\
DA4 & $11,860-13,340$ & $4.25-3.75$ \\
DA4.5 & $10,610-11,860$ & $4.75-4.25$ \\
DA5 & $9,600-10,610$ & $5.25-4.75$ \\
DA5.5 & $7,467-9,600$ & $6.75-9.25$ \\
DA6 & $6,952-7,467$ & $7.25-6.75$ \\
DA6.5 & $6,503-6,952$ & $7.75-7.25$ \\
DA7 & $6,109-6,503$ & $8.25-7.75$ \\
DA7.5 & $5,760-6,109$ & $8.75-8.25$ \\
DA8 & $5,448-5,760$ & $9.25-8.75$ \\
DA8.5 & $5,169-5,448$ & $9.75-9.25$ \\
DA9 & $4,917-5,169$ & $10.25-9.75$ \\
DA9.5 & $4,688-4,917$ & $10.75-10.25$ \\
DA10 & $4,480-4,688$ & $11.25-10.75$ \\
DA10.5 & $4,289-4,480$ & $11.75-11.25$ \\
DA11 & $4,114-4,289$ & $12.25-11.75$ \\
DA11.5 & $3,952-4,114$ & $12.75-12.25$ \\
DA12 & $3,803-3,952$ & $13.25-12.75$ \\
DA12.5 & $3,665-3,803$ & $13.75-13.25$ \\
DA13 & $3,537-3,665$ & $14.25-13.75$ \\
\hline \hline
\end{tabular}

\subsubsection{Symbols for White Dwarfs with Extreme Surface Gravity}

We have introduced optional symbols for white dwarfs showing very diffuse, broad lines corresponding to exceptionally high gravity (log $g \gtrsim 9$ ) and white dwarfs with narrow sharp lines of exceptionally low gravity $(\log g \lesssim 7$ ). The former are denoted by a lower case $\mathbf{d}$ for diffuse, broad lines while the latter are denoted by lower case $\mathbf{n}$ for sharp, narrow lines. These symbols should be assigned only where spectroscopic or dynamical gravity determinations are ironclad indicators of extremely high or extremely low gravity. 


\subsubsection{Classification of the Hottest Non-DA Stars}

The hottest non-DA stars present problematic classification because (1) many are planetary nebula nuclei and isolated post-AGB stars sharing the hallmark spectroscopic characteristics of the PG1159 degenerates but their gravities are lower than $\log g=7$ which is traditionally adopted as the minimum defining gravity for classification as a white dwarf star (versus a high gravity subdwarf; Greenstein \& Sargent 1974) and; (2) the assignment of the primary spectral class for a white dwarf is determined by the element represented by the strongest absorption features in the optical spectrum. However, by this criterion, PG1159 stars, for which either oxygen (e.g., O VI) or carbon (e.g., C IV) are the strongest optical lines (with He II features weaker), should be classified DZQO or DQZO depending upon whether O VI or C IV are strongest, respectively. Since many of these objects have atmospheric compositions which are not helium-dominated (cf., Werner \& Heber 1992), it is inappropriate to assign spectral type DO on the Sion et al. (1983) scheme since the primary O-symbol denotes a heliumdominated composition. Here we adopt the primary spectroscopic type as the atom or ion with the strongest absorption features in the optical spectrum, where applicable (for example, it is possible that the strongest absorption feature may lie in the space ultraviolet). This scheme for classifying the hottest degenerates preserves consistency with the original classifications of white dwarf spectra, which have been based upon what absorption features are actually seen in the optical spectra (cf. Greenstein 1960; Sion et al. 1983).

In this edition we have withheld a degenerate classification for any PG1159 star with $\log g<7$. However, these objects are designated PG1159 in the spectral class entry and the types, as given by Werner \& Heber (1992) and subsequently used by Dreizler, Werner \& Heber (1995) and Napiwotzski \& Schonberner (1991), are given in the notes section of this catalog. These designations are: E for emission, lgE for low gravity, A for absorption, Ep for emission/peculiar, EH, lgEH or AH for hybrid PG1159s which have detected H.

The temperature index would differentiate the hot C-He-O stars from the well-known, very much cooler DZ and DQ degenerates below 10,000K. The temperature index of the PG1159 stars would be (as for all white dwarfs) given by $10 \times \theta_{\text {eff }}\left(=50400 / \mathrm{T}_{\text {eff }}\right)$. For example PG1159 itself $\left(\log g=7, \mathrm{~T}_{\text {eff }}=\right.$ 110,000K, C IV absorption strongest optical lines) would be classified DQZO.4. The obvious disadvantage is the inevitable confusion with the cool DQ and DZ degenerates in cases where the temperature index is missing or there are no He II absorption features (e.g., H1504). In a case like H1504 where no helium is detected and $\mathrm{T}_{\text {eff }}=170,000 \mathrm{~K}, \log g=7$, our classification scheme would assign a spectral class DZQ.3. 


\subsubsection{Binary Membership Designation}

All binary systems containing one or two degenerate stars (and essentially noninteracting), regardless of orbital separation, are denoted by lower case "b" in the column just preceding the start of the reference number of the spectral type assignment. Thus these would range from very close non-interacting pairs (e.g., pre-cataclysmics) to common proper motion systems containing white dwarfs and having no measurable orbital motion.

\subsection{Color- $\mathbf{T}_{e f f}$ Relations and Color-Absolute Magnitude Correlations}

The color-effective temperature relations are the same as those employed by McCook \& Sion (1987), which were adopted from Shipman (1979). Temperaturecolor index correlations using model atmospheres for DA and non-DA stars are available for multichannel spectrophotometric colors $g-r$; Strömgren colors $(u-b$, $b-y)$; and broad-band $U B V$ colors $(B-V, U-B)$. Temperatures derived from different model atmosphere grids are generally very consistent. We determine the temperature index by using the color transformations based upon Tables 1 and 2 and equations (9) and (10) of Shipman (1979). The transformation relations for non-DA stars are:

$$
\begin{gathered}
(B-V)=0.334+0.836(g-r), \\
(b-y)=0.286+0.553(g-r) .
\end{gathered}
$$

For DA stars the color transformations are

$$
(B-V)=0.3336+0.5906(g-r),(b-y)=0.2197+0.4486(g-r) .
$$

Similarly, the color-absolute magnitude correlations are presented in McCook \& Sion (1987) are adopted in this edition. The original references to these calibrations are Green (1977), Greenstein (1984), Sion \& Liebert (1977), and Dahn et al. (1982). The same priortized system of photometric parallaxes adopted by McCook \& Sion (1987) is used in this edition, including the use of trigonometric parallax when multiple values of the parallax yield a mean $>0.100$ arc-seconds. An absolute visual magnitude has been computed for each star with measured colors or trigonometric parallax according to the system described below. Exceptions to this system are denoted with numerical codes $(0),(5),(6),(7),(8)$ and (9) and are also described below. If measured colors do not exist, an absolute magnitude was not given. For all spectral types with $(g-r)$ color index the following calibration formula due to Greenstein (1984) was employed:

$$
M_{v}=13.033+3.114(g-r)-0.799(g-r)^{2} .
$$

For all spectral types with $(b-y)$ color index, the following calibration due to Green (1977) was adopted: 


$$
M_{v}=7.56(b-y)+11.50 .
$$

The broad-band calibrations are those of Sion and Liebert (1977). For DA white dwarfs with $B-V<0.4$,

$$
M_{v}=11.246(B-V+1)^{0.60}-0.045 .
$$

For non-DA stars with $B-V<0.4$,

$$
M_{v}=11.916(B-V+1)^{0.44}-0.011 .
$$

If $B-V \geq 0.4$, we used the following color-magnitude calibration due to Dahn et al. (1982):

$$
M_{v}=(11.43 \pm 0.16)+(7.25 \pm 0.75)(B-V)-(3.42 \pm 0.68)(B-V)^{2} .
$$

The color-magnitude relations defined above are fairly accurate and are the same as we used in the third edition. However, other relations based upon colormagnitude predictions from model atmospheres (and fitting the observed data) are even more accurate. In particular we refer the user to Bergeron, Wesemael and Beauchamp (1995).

In a few cases where three or more measured parallaxes from different sources are in agreement, exceptions were made to the priority system above. Likewise, for degenerate stars whose spectra exhibit abnormally strong or peculiar blanketing (e.g., G 47-18, GD 229, LP 701-29) and for every cool white dwarfs (e.g., LP 131-66), the above priority system was not followed. Since for cool white dwarfs the blue colors (e.g., $B-V, b-y, g-r$ ) yield less accurate photometric parallaxes than the red colors (e.g., $V-I, R-I)$, we have adopted absolute magnitudes from four sources: (a) the tabulation of cool white dwarfs by Liebert, Dahn, \& Monet (1987) if our values differed by more than 0.3 mag from theirs; (b) the extensive compilation of absolute magnitudes for cool white dwarfs by Bergeron, Ruiz and Leggett (1997); (c) the values for Palomar -Green DA stars utilized Liebert et al. (1997) and; (d) the compilation of absolute magnitudes for the subset of common proper motion binaries containing white dwarfs which were used to derive the white dwarf luminosity function in the dissertation by Smith (1998). The absolute magnitude values from these three are denoted by numerical codes (6), for Liebert, Dahn and Monet (1987), (7) for Bergeron, Ruiz and Leggett, and (8) for Liebert et al. 1997, and (9) the values in Smith's (1998) $\mathrm{Ph} . \mathrm{D}$ thesis. An extensive tabulation of red colors of white dwarfs can be found in a review by Eggen (1985 and references therein), while $V$ - $I$ color indices are available from the US Naval Observatory faint star parallax lists cited in this catalog. Likewise, for the very hot DA stars where the blue-sensitive colormagnitude calibrations are insensitive to temperature, we have used the values of $M_{v}$ derived from actual effective temperature determinations as given, for example, by Fleming, Green, \& Liebert (1986) and Holberg, Wesemael, \& Basile 
(1986). Here again, however, their values were adopted only if a discrepancy greater than 0.3 mag existed. These values are denoted by numerical code (0). For the hottest helium-rich degenerates the photometric parallaxes are grossly inaccurate. For the DO and DOZ white dwarfs, we have replaced the $M_{v}$-value with the directly derivable effective temperature in degrees Kelvin. Most of the adopted values were from Wesemael, Green, \& Liebert (1985), Werner \& Heber (1992), Dreizler et al. (1995) and Napiwotzki and Schonberner (1991), and are indicated by numerical code (5). A discussion of the dispersions and analysis of the calibrations is given in Sion (1979) and Sion (1984). The value of absolute visual magnitude is followed by number $1,2,3$, or 4 to indicate the method of determination; (1) parallax, (2) multichannel color, (3) Strömgren color, and (4) $U B V$ color, or the value is followed by (0), (5), (6), (7), (8) and (9) as described earlier. Stars with hybrid classifications were generally not assigned a photometric parallax.

The advent of increasingly accurate effective temperatures and gravities from detailed spectroscopic fits for DA stars (cf., Bergeron, Saffer \& Liebert 1992; Finley 1993; Kidder 1992) and gravities (Bergeron, Saffer \& Liebert 1992), for DB stars (Thejll et al. 1991), and the coolest non-DA degenerates, has made it possible to re-generate more precise photometric parallaxes and temperature indices for a large number of degenerates subject to the following caveat. Unless the white dwarf mass is known, there will be a non-negligible uncertainty in the white dwarf luminosity. The appropriate reference is given immediately following the spectral type. See Table 3 at the end of the paper.

\section{THE ENTRIES IN TABLE 3}

All entries in Table 3 except coordinates, names, and absolute magnitudes are followed by a reference number in parentheses - e.g., (41). The Reference Key, which follows the Notes, can then be used to identify the source of the catalog entries.

WD. - The first entry contains a "WD number", a catalog number containing the first four digits of right ascension followed by the sign, the first two digits of declination, and third digit in which minutes of declination have been expressed as a fraction of a degree. Stars with identical catalog numbers, whether binary or not, have been distinguished by using the designations .1 and .2 after their respective catalog numbers. The same distinction is used for wide binary pairs. The choice of the WD number format was made with the idea of continuing expansion of the catalog as data accumulate.

R.A. DEC. - The second entry contains the full right ascension and declination (1950.0) adopted according to the following order of priority: Lowell Observatory coordinates, Luyten Proper Motion Survey coordinates. For Bruce Proper Motion Survey stars, the coordinates may refer to epoch and 
equinox 1900.0 if no other proper motion name is listed. In relatively few cases cooordinates refer to epoch and equinox 2000.0. These coordinates are indicated with a "J" appended to the WD number.

SPEC. CLASS. - The third entry lists the spectral type according to the system described by Sion et al. (1983), updated with the modifications described in section 2 where a complete discussion may be found. The definition of primary spectral symbols is given in Table 1 according to the Sion et al. (1983) classification. If more than one spectral class assignment has been made and cited and they are in agreement, the spectral type has been converted to the new system and is followed by the original reference(s). If more than one spectral class has been cited and they are in disagreement, a spectral type converted to the new system is given based upon our assessment of the spectral resolution and instrumental technology involved, but the old spectral types are retained followed by their references. In many cases the primary classification reference has not been listed but may be easily found in the secondary reference which is given. A lower case "b" is inserted just before the spectral type reference if the white dwarf is a member of a non-interacting binary (see section 2.1.4). An asterisk $\left(^{*}\right)$ is inserted if an entry exists in Notes to Table 3 for white dwarfs of exceptional astrophysical interest.

NAMES. - The fourth entry contains most of the names that exist for each star. The names are listed below, with most of their references. An extensive list of cross-references to the names in Table 3 can be found in Table 4 .

\begin{tabular}{|ll|}
\hline BPM: & - Bruce Proper Motion Survey: see Reference Key under \\
& Luyten. \\
C: Case: & - Stephenson $(1960,1962)$. \\
C120: Cincinnati: & - Porter, Yowell, \& Smith (1930). \\
CSO,CBS: & - Case Low Dispersion Survey: Pesch \& Sanduleak \\
& $(1983,1985)$, \\
& Sanduleak \& Pesch (1984). \\
CTI: & - CCD Transit: Kirkpatrick \& McGraw (1989). \\
DeHt: & - Planetary nebula name, authors' initials (Dengel et \\
& al.): \\
& Perek \& Kohoutek (1967). \\
EC: & Edinburgh-Cape Survey: see Reference Key under \\
& Stobie et al. and references therein. \\
EG: & Eggen-Greenstein: see Reference Key under Eggen \& \\
& Greenstein. \\
EUVE: & - Newly Identified white dwarf with the Extreme Ultra- \\
& violet Explorer: \\
& see Reference Key under Vennes.
\end{tabular}




\begin{tabular}{|c|c|}
\hline F, Feige: & - Feige (1958). \\
\hline $\mathrm{G}, \mathrm{GD}, \mathrm{GH}:$ & - Lowell names: see Reference Key under Giclas. \\
\hline GL: Gliese: & - Gliese (1957). \\
\hline GR: Greenstein: & - See Reference Key under Greenstein. \\
\hline GW: Greenwich: & - Dyson (1914). \\
\hline HaWe: & $\begin{array}{l}\text { - Planetary nebula name, authors' initials, Acker } \\
\text { (1992). }\end{array}$ \\
\hline HDW: & $\begin{array}{l}\text { - Planetary nebula name, authors' initials, Perek \& Ko- } \\
\text { houtek (1967). }\end{array}$ \\
\hline HE: & - Hertzsprung: Hertzsprung (1918). \\
\hline $\mathrm{HE}$ : & - Hamburg-Schmidt Survey (southern hemisphere). \\
\hline HL: & - Tonantzintla: Haro \& Luyten (1960). \\
\hline HS: & $\begin{array}{l}\text { - Hamburg-Schmidt Objective Prism Survey: see Ref- } \\
\text { erence Key } \\
\quad \text { under Heber, Napiwotzski, Jordan, Dreizler. }\end{array}$ \\
\hline HZ: & - Humason-Zwicky: Humason \& Zwicky (1947). \\
\hline IsWe: & $\begin{array}{l}\text { - Planetary nebula name, authors' initials, Acker } \\
\text { (1992). }\end{array}$ \\
\hline IW: & $\begin{array}{l}\text { - Planetary nebula name, authors' initials, Acker } \\
\text { (1992). }\end{array}$ \\
\hline $\mathrm{K} 1:$ & - Kohoutek (K1̈̈ 16): Perek \& Kohoutek (1967). \\
\hline Karpov (K1-K12): & - Karpov (1937). \\
\hline KUV: & $\begin{array}{l}\text { - Kiso: see References under Kondo et al. }(1982,1984) \text {, } \\
\text { Noguchi et al (1980). }\end{array}$ \\
\hline KPD: Kitt Peak-Downes: & - Downes $(1986)$ \\
\hline $\begin{array}{l}\text { L, LB, LP, LDS, LHS, LTT: } \\
\text { LBQS: }\end{array}$ & $\begin{array}{l}\text { - Luyten names: see Reference Key under Luyten. } \\
\text { - The Large Bright Quasar Survey: see Reference Key } \\
\text { under Hewett, } \\
\text { Foltz. }\end{array}$ \\
\hline M, Mn- : & - Planetary nebulae, Minkowski (1948), Acker (1992). \\
\hline MCT: & $\begin{array}{l}\text { - Montreal-Cambridge-Tololo Survey: see Reference } \\
\text { Key under } \\
\text { Wesemael, Fontaine, Lamontagne. }\end{array}$ \\
\hline MK: & $\begin{array}{l}\text { - Markarian: see References under Markarian \& } \\
\text { Lipovetski (1971). }\end{array}$ \\
\hline Other: Other names: & - Fernandez et al. (1983). \\
\hline PB: & - Palomar-Berger: Berger \& Fringant (1977). \\
\hline PG: & $\begin{array}{l}\text { - Palomar-Green: see References under Green, Schmidt, } \\
\text { \& Liebert. }\end{array}$ \\
\hline PHL: Tonantzintla: & - Haro \& Luyten (1962). \\
\hline R: Ross: & - Ross (1925-1939). \\
\hline RB, RWT: & - Rubin: Rubin,Westphal, \& Tuve (1974). \\
\hline RE: & $\begin{array}{l}\text { - ROSAT Wide Field Camera Survey: see Reference } \\
\text { Key under Barstow. }\end{array}$ \\
\hline SA: & - Basle Halo Program: Steppe (1978). \\
\hline SB: & - see References under Slettebak \& Brundage (1971). \\
\hline TC, TON, TPS, TS: & $\begin{array}{l}\text { - Tonantzintla: Iriarte \& Chavira (1957), Chavira } \\
\text { (1959), } \\
\text { Philip \& Sanduleak (1968). }\end{array}$ \\
\hline US: Usher: & - Usher (1981). \\
\hline VB: Van Biesbroeck: & - Van Biesbroeck (1961). \\
\hline V Ma: Van Maanen: & - Van Maanen (1938). \\
\hline VR: Van Rhijn-Raimond: & - Van Rhijn \& Raimond (1934). \\
\hline W: Wolf: & - Wolf (1919-1929). \\
\hline
\end{tabular}


WIDE BAND. - $(V, B-V, U-B)$. The fifth entry contains published values of wide-band $U B V$ photometry. The $V$-magnitude is listed followed by $B-V, U$ $B$, and the reference number. If the star has not been observed on the $U B V$ system, a photographic magnitude and color class (CC) is given from either the Lowell or Luyten Proper Motion Survey. Photographic magnitudes are indicated by a $\mathrm{PG}$ following the magnitude in the $\mathrm{V}$ column.

MC $(V, g-r)$. - The sixth entry contains multichannel spectrophotometric observations. The multichannel $V$-magnitude is listed, followed by multichannel color and the reference.

STRÖMGREN $(y, b-y, u-b)$. - The seventh entry lists the Strömgren, narrow-band, photometry, $y, b-y, u-b$, and the reference.

ABS. MAG. - The eighth entry lists the absolute visual magnitude computed from either trigonometric parallax, measured colors, or taken from published compilations based upon red photometry. Note that in the case of DO, DOZ and PG1159 stars, the absolute magnitude is replaced by a spectroscopically determined Teff. The absolute magnitude entry is followed by a single digit numerical code to indicate the method of calculation or the source: (1) trigonometric parallax $>0 " 1,(2)$ multichannel spectrophotometric colors, (3) Strömgren narrow-band colors, (4) UBV photometry, (5) Teff value from Wesemael, Green and Liebert (1985), Werner and Heber (1992), Dreizler et al. (1995), or Napiwotzki \& Schonberner (1991), (6) Liebert, Dahn and Monet (1988), (7) Bergeron, Ruiz and Leggett (1997), (8) Liebert et al. 1997, and (9) Smith (1998).

MU, THETA - The ninth entry contains the published values of proper motion and position angle followed by the reference. The units are seconds of arc per annum for proper motion, and degrees for the position angle.

VRO. - The tenth entry lists the observed radial velocity uncorrected for solar motion and gravitational redshift and followed by the reference.

PI, ME. - The final catalog entry lists the published values of the trigonometric parallax in seconds of arc. In most cases the parallax is followed by the reported mean error in units of 0.001 .

We are deeply indebted to the following colleagues for communicating survey data in advance of publication, providing updated or revised spectral types or other crucial information needed for the production of this edition: Martin Barstow, Pierre Bergeron, Paul Bradley, Conard Dahn, Gilles Fontaine, Uli Heber, Jay Holberg, R. Lamontagne, Barry Lasker, Jim Liebert, Terry Oswalt, Darragh O'Donoghue, Rex Saffer, Gary Schmidt, Allyn Smith, Richard Tweedy, Bill van Altena, Stephane Vennes, Volker Weidemann, Klaus Werner, and Francois Wesemael. At Villanova it is a pleasure to thank Samir Bham, David Steelman, Srinivas Narendula, Shami Reddy, Gopi Gopivallabha, Radhika Krishnan, Kunegunda Belle, Qing Sung, Rick Wasatonic and Mai Huang for their patient and careful assistance with data compilation and proofread- 
ing. We gratefully acknowledge the support of the National Science Foundation through grants AST88-17172 and AST90-16283.

\section{REFERENCES}

A. Acker, A. et al. 1992, Strasbourg-ESO Catalogue of Galactic Planetary Nebulae (Garching bei Munchen, ESO)

Berger, J., \& Fringant, A. M. 1977, A\&AS, 28, 123

Bergeron, P., Saffer, R., \& Liebert, J. 1992, ApJ, 394, 228

Bergeron, P., Wesemael, F. \& Beauchamp, A. 1995, PASP, 107, 1047.

Bergeron, P., Ruiz, M.-T., \& Leggett, S.K.1997, ApJS

Chavira, E. 1958, Bol. Obs. Tonantzintla y Tacubaya, 3, No. 17

Chavira, E. 1959, Bol. Obs. Tonantzintla y Tacubaya, 3, No. 18

Dahn, C. C., et al. 1982, AJ, 87, 426

Downes, R. 1986, ApJS, 61, 569

Dreizler, S., Werner, K., \& Heber, U.1995, in White Dwarfs, eds. D. Koester \& K. Werner, (Springer: Berlin), 160

Dyson, F. W. 1914, Astrographic catalogue, 1900.0 greenwich section, Vol. 3, +64 to + 90, (Edinburgh: Royal Observatory, Greenwich)

Eggen, O. J. 1985, PASP, 97, 1029

Feige, J. 1958, ApJ, 128, 267

Fernandez, A., Lortet, M.-C., \& Spite, F. 1983, A\&AS, 52, No. 4

Finley, D. 1993, in White Dwarfs: Advances in Observations and Theory, ed. M. A. Barstow (Kluwer, Dordrecht)

Fleming, T., Green, R. F. \& Liebert, J. 1986, ApJ, 308, 176

Green, R. F. 1977, Ph.D. thesis, California Institute of Technology

Green, R. F., Schmidt, M., \& Liebert, J. 1986, ApJS, 61, 305

Greenstein, J. L. 1960, in Stellar Atmospheres, Stars and Stellar Systems, ed. J. L. Greenstein (Chicago: University of Chicago), Vol. 6, p.676

Greenstein, J. L., \& Sargent, W. 1974, ApJS, 28, 157

Greenstein, J. L. 1984, ApJ, 276, 602

Greenstein, J. L. 1986, ApJ, 304, 334

Haro, G. \& Luyten, W. J. 1960, Bol. Obs. Tonantzintla y Tacubaya, 29, 16

Haro, G. \& Luyten, W. J. 1962, Bol, Obs. Tonantzintla y Tacubaya, 3, No. 22

Hertzsprung, E. 1918, AstrNach, 207, 171

Holberg, J. B., Wesemael, F., \& Basile, J. 1986, ApJ, 306, 629

Humason, M. \& Zwicky, F. 1947, ApJ, 105, 85

Iriarte, B. \& Chavira, E. 1957, Bol. Obs. Tonantzintla y Tacubaya, 3, No. 16

Karpov, B. G. 1937, PASP, 49, 146

Kidder, K. 1992, Ph.D Thesis, University of Arizona

Kirkpatrick, J.D., \& McGraw, J. T. 1989, in White Dwarfs, (IAU Colloq. No. 114), ed. G. Wegner, (Berlin: Springer), 167

Kondo, M., Noguchi, T., \& Maehara, H. 1984, Ann. Tokyo Astr. Obs., Ser. 2, 20,130

Kondo, M., Watanabe, E., Yutani, M., \& Noguchi, T. 1982, PASJ, 34, 541 
Liebert, J., Dahn, C. C., \& Monet, D. G. 1988, ApJ, 332, 891

Liebert, J., \& Sion, E.M. 1994, in The MK Process at 50 Years: A Powerful Tool for Astrophysical Insight, (PASP Conf. Series: 60), p.64

Liebert, J., Fleming, T., Holberg, J., Bergeron, P., \& Saffer, R.1997, in "White Dwarfs", eds.J.Isern, M. Hernanz \& E. Garcia-Berro, (Dordrecht: Kluwer), p.85.

Markarian, B. E. \& Lipovetski, V. A. 1971, Astrofizika, 7, 511

McCook, G. P., \& Sion, E. M. 1977, A Catalogue of Spectroscopically Identified White Dwarfs (1st ed.; Villanova Univ. Obs. Contr., No. 2)

McCook, G. P., \& Sion, E. M. 1983, A Catalogue of Spectroscopically Identified White Dwarfs (2nd ed.; Villanova Univ. Obs. Contr., No. 3)

McCook, G. P., \& Sion, E. M. 1987, ApJS, 65, 603

Minkowski, R. 1948, PASP, 60, 386

Napiwotszki, R., \& Schonberner, D. 1991, in White Dwarfs, ed. G. Vauclair \& E. M. Sion (NATO ASI Series, Series C, 336: Dordrecht, Kluwer), p.39

Noguchi, T., Machara, H., \& Kondo, M. 1980, Ann. Tokyo Astr. Obs., Ser. 2, 18,55

Perek, L. \& Kohoutek, L. 1967, Catalogue of Galactic Planetary Nebulae, (Czech. Acad. Sci: Prague), 267

Pesch, P., \& Sanduleak, N. 1983, ApJS, 51, 171

Pesch, P., \& Sanduleak, N. 1985, ApJS, 60, 543

Philip, A. G. D., \& Sanduleak, N. 1968, Bol. Obs. Tonantzintla y Tacubaya, 4, No. 30

Porter, J. G., Yowell, E.J. \& Smith, E.S. 1930, Pub. Cincinnati Obs., No. 20

Ross, F. E. 1925-1939, AJ, Vols. 36-48

Rubin, V. C., Westphal, D., \& Tuve, M. 1974, AJ, 79, 1406

Sanduleak, N., \& Pesch, P. 1984, ApJS, 55, 517

Shara, M., Bergeron, E. Christian, C.A., Craig, N., \& Bowyer, S. 1997, PASP, in press

Shipman, H. L. 1979, ApJ, 228, 240

Sion, E. M. 1979, IAU Colloquium 53, White Dwarfs and Variable Degenerate Stars, ed. H. M. Van Horn \& V. Weidemann (Rochester: University of Rochester Press), p. 245

Sion, E. M. 1984, ApJ, 282, 612

Sion, E. M., Greenstein, J. L., Landstreet, J. D., Liebert, J., Shipman, H. L., \& Wegner, G. A. 1983, ApJ, 269, 253

Sion, E. M., \& Liebert, J. 1977, ApJ, 213, 468.

Sion, E.M. 1996, in Hydrogen Deficient Stars and Related Objects, eds. S. Jeffrey \& U. Heber, (PASP Conf. Ser.), in press

Slettebak, A. \& Brundage, R.K. 1971, AJ, 76, 338

Smith, J.A. 1998, Ph.D Thesis, Florida Institute of Technology, Melbourne, FL.

Stephenson, C. B. 1960, PASP, 72, 387

Stephenson, C. B. 1962, PASP, 74, 610

Steppe, H. 1978, A\&AS, 31, 209 
Stobie, R.S., Chen, A., O'Donoghue, D., Kilkenny, D.1992, in "Variable Stars and Galaxies", ed. B. Warner, PASP Conf. Series, 30, 87

Thejll, P., Vennes, S., \& Shipman, H.L. 1991, ApJ, 370, 355

Usher, P. D. 1981, ApJS, 46, 117

Van Biesbroeck, G. 1961, AJ, 66, 528

Van Maanen, A. 1938, ApJ, 88, 27

Van Rhijn, P. J. \& Raimond, J. J. 1934, MNRAS, 94, 508

Von Gliese, W. 1957, Katalog der Sterne Naher als 20 parsek für 1950.0 (Heidelberg)

Werner, K., \& Heber, U. 1992, in The Atmospheres of Early Type Stars, ed. U. Heber \& S. Jeffrey (New York, Springer), p. 273

Wesemael, F., Green, R. F., \& Liebert, J. 1985, ApJS, 58, 379.

Wesemael, F., Greenstein, J. L, Liebert, J., Lamontagne, R., Fontaine, G., Bergeron P., \& Glaspey, J. W. 1993, PASP, 105, 761

Wolf, M. 1919-1929, Veröff Sternwarte zu Heidelberg, 7, No. 10 (Astr. Nach., 209-236) 